\title{
Design of A Simple UV Double Beam Spectrophotometer Detector Based on a High Gain Trans Impedance Operational Amplifiers for RNA Measurement
}

\author{
http://dx.doi.org/10.3991/ijes.v4i2.5905 \\ Alakhib I. Abdelbary ${ }^{1}$, Abdelrahim Elobied Ahmed ${ }^{2}$ \\ ${ }^{1}$ Central Laboratory, Khartoum, Sudan \\ ${ }^{2}$ Sudan International University, Khartoum, Sudan
}

\begin{abstract}
In this work a simple double beam spectrophotometer detector for the nucleic acid detection has been designed. The developed system contains photodiodes as a sensor, logarithamatic transimpedance amplifier circuit and filter circuit.The developed prototype design accuracy is validated by running a RNA sample and the result shows that our simplified developed setup detects the present of RNA in the sample.
\end{abstract}

Index Terms-Trans impedance amplifier, RNA, double beam, Photodiode.

\section{INTRODUCTION}

Ribonucleic acid (RNA) is a polymer molecule [1] implicated in various biological roles in coding, decoding, regulation and expression of genes, RNA is assembled in as a chain of nuletidedes, it is structure contains, a ribose sugar with carbon, adenine, cytosine and gulanine or uracil. The function of RNA is carrying out the instructions encoded in DNA, there are three kinds of RNA first messenger RNA (mRNA), second transfer RNA (tRNA) and ribosomal RNA(rRNA)[2].

There are different detection methods for RNA such as cell culture techniques, nucleic acid amplification based technique, absorbance and fluorescence techniques[3].

The detection of RNA is applied in various fields such as diagnosis of diseases (infectious and hereditary) [18][19] pathogenic detection [20] detection of biological wafer agent [21].The detection of RNA also allows the doctors and scientist to get answers about the type of virus and bacteria cell behind the infectious diseases[22].

There are a different types of biosensors using in the sensing of the RNA, such as using the amperometeric biosensor detect the RNA by facilitating sandwich hybridization test of nucleic acid (DNA, RNA) [23] as results of interacting, a carbon electrode with modified film. The detection of RNA based on different types of techniques such as field effect device, nanowire, carbon nanotubes[24] [25]. N.Guo described CMOS imaging device for nucleic acid detection in [26].

Electron microscopy permits the determination of the length of RNA and DNA [28]. Real time PCR is a recent modifies which combines the objective fluorescence detection with the basic PCR assay; use to obtain multiple copies of DNA fragments of sample contain multiple copies of DNA. Fragment from sample contain minute quantization of DNA or RNA [29].

The spectrophotometer is one of the accurate instruments used for the quantization of RNA, it is the instrument used to measure the intensity of light as a function of it is the wavelength. The first measurement of nucleic acid quantization is done by Christian and Warburg [15].

The main challenges facing the detection of RNA include high sensitivity, specificity and protocols that conduct the analysis in a short time with low detection limit [30] and low volume.There are many problems facing the performance of the spectrophotometer systems mainly from the detector circuit such as dark current for the sensor and the oscillations that caused by a capacitor diode form the photodiode on the input is other sources of noise in addition to preamplifier circuit problems such as the input offset voltage, temperature variations and the input bias current.

The main objective of this work is to make a simple design that increase sensitivity of the absorbance spectrophotometer detector to detect the RNA sample without adding dyes or other flours material.

The work is organized as follows: section II illustrates the system description. The proposed design setup and components selection is described in section III, section IV presents the results and discussion and the conclusion is reported in section $\mathrm{V}$.

\section{SYSTEM DESCRIPTION}

The block diagram shown in fig.1 depicts a general double beam spectrophotometer system. The main system components are light source as which generates light in the entire spectrum range to cover the UV wavelength radiation.the light pass through mirror1 (focusing mirror) to grating device which used to disperse the light according to their wavelengths. Mirror 2 accumulates the light and pass it to the Spilitting mirror which divert the light to the Sample and reference end points.

There are different types of sensors used for the measuring of radiation energy, such as photovoltaic cell barrier, a high vacuum photo emissive cell and the photomultiplier tube and photodiode. The photomultiplier tube is most commonly photon emissive cell which has been used largely in the spectrophotometer and fluorescence spectroscopy, it is very sensitive to low intensity of light [9]. 
Photodiode also used largely in photometer circuits as a detector [10]. Is the one of the most popular types for many lights based measurement applications such as absorption and emission spectrophotometer devices [11].The selection of the operational amplifier for the spectrophotometer detector circuit play vital role in the design of the spectrophotometer circuit, therefore a lot of operational amplifiers used for the converted of the current to voltage such as a chopper stabilized amplifier, lock amplifier and transimpedance amplifier.

\section{The Proposed Design SETUP And Components SELECTION}

This experimental design setup were carried out to develop a simple detector for the double beam spectrophotometer based on the logarithmtic transimpedance amplifier to acquire signal from spectrometer (optical system) as described in Fig.2. The light from UV deuterium lamp (L2D2) enter the spectrometer and incidance on the entrance slit to the collimating mirror, and passes to the differaction grating. The diffracted light focused by a second mirror on the optical beam splitter to passes through exit slit to the reference and sample cuvette.

The function of photodiode as stated before is the conversion of light to electricity, the specifications of our photodiode 1227 as below: the photosensitive area of the photodiode is $2.4 \times 2.4 \mathrm{~mm}$ and noise equivalent power

$2.5 \times 10 e^{-15}$ ? The equivalent photodiode circuit contains diode, it's the semiconductor P.N. Junction, the junction capacitance $\mathrm{Cj}$ and a shunt resistance Rsh. As shown in figure. 3 the conversion of current to voltage using photodiode is described by [12].

The two photodiode recived the light from the sample and reference as shown in the fig. 3

To verify the linearity property of the detector, the light on the photodiode is closed step by step until closed completely, in every step the current and the output voltage have been measured this is done by using digital multimeter type fluke 179 true rms multimeter and oscilloscope GOS-658G 50MHz.

\section{A. Transimpedance amplifier circuit}

When the light incidence on the photodiode, the photodiode(refer to fig 4) generate the output current according to incidence light, for these experiments the MAX4206 high precision transimpedance log amplifier from Maxim company, used to compute the log ratio of input current from the reference and sample photodiodes as described by beer Lambert law[16]by the equation as below:

$$
A=\log \left(I / I_{0}\right)
$$

Where $\mathrm{A}$ is the absorbance, $\mathrm{I}$ is the sample current and $1^{\mathrm{o}}$ is the reference current. As shown in fig. 3 .

The Max4206 provide a corresponding voltage output as given by the equation:

$$
V_{\text {out }}=k \log \left(I / I_{0}\right)
$$

Where $\mathrm{k}$ is $\mathrm{KT} / \mathrm{q}, \mathrm{K}=$ boltzman constant is $1.38 \times 10^{-23} \mathrm{~J} / \mathrm{K}, \mathrm{T}=$ Absolute temperature and

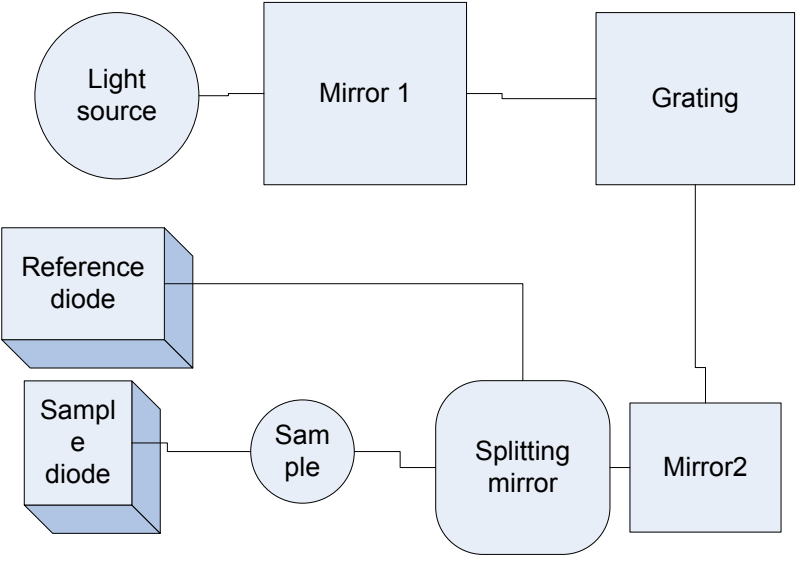

Figure 1. block diagram of double beam spectrophotometer

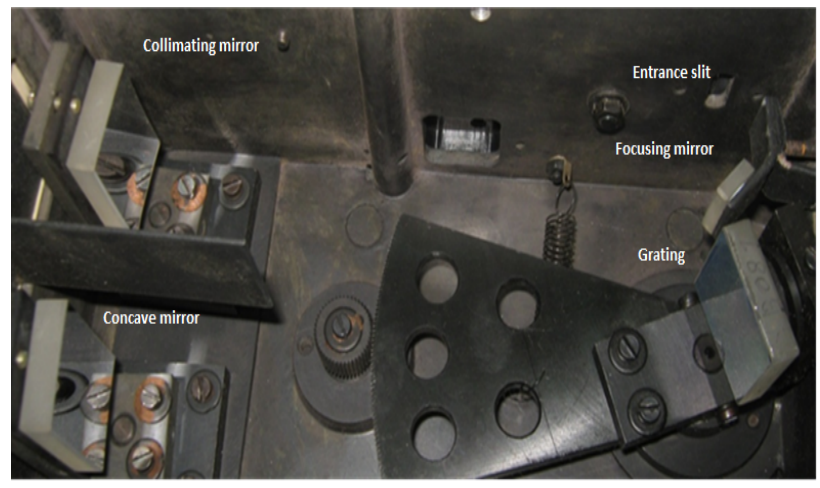

Figure 2. Picture of the optical system based on the Czenry turner configuration

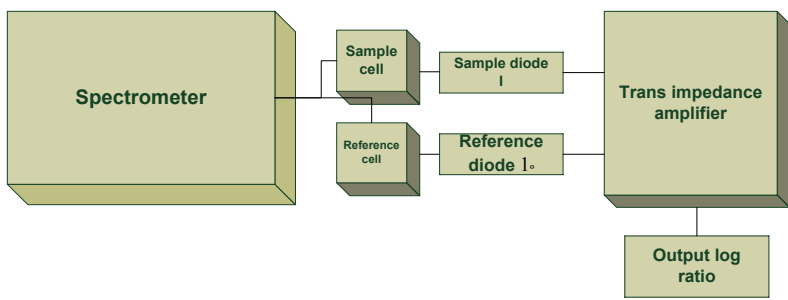

Figure 3. The block diagram of the system setup.

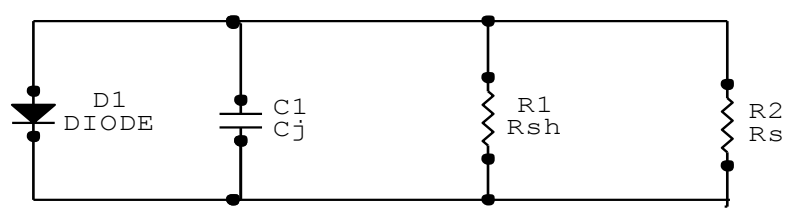

Figure 4. Equivalent circuit of silicon photodiode.

$q=1.60210^{-19 \circ} \mathrm{C}$. The Max4206 integral logarithmic amplifier is used for two purposes convert current to voltage and express and output that is related to it is input by mathematical[4].

The circuit of the photodiodes and the transimpedance amplifier circuit shows in fig.5, it contains MAX4206 operatin amplifier, two photodiodes sample diode (D1), and reference diode (D2). The resistors R1 and R2 equal to $100 \Omega$ functions as a load resistance element for the photodiode, C2 and C3 100pF capacitor has been used for stabilization and compensation of the input capacitance of the photodiodes, $100 \mathrm{pF}$ resulted in close loop bandwidth in the range of $\mathrm{KHz}$ as a typical of many spectroscopy 
PAPER

Design of A Simple UV Double Beam Spectrophotometer Detector Based on a High Gain...

systems[17].The $0.1 \mu \mathrm{F}$ capacitor $(\mathrm{C} 1)$ used on the input of the preamplifier to eliminate high frequency noise produced by a resistor.

The stable bandwidth attainable with a transimpedance amplifier gain of $5 \mathrm{MHz}$ and feedback resistor $\mathrm{Rf}$ of $30 \mathrm{k} \Omega$. The dominated noises encountered in the detector are resistors noise referred as Johnson noise generated due to resistance material and bandwidth, the input voltage noise generated from dark current, shot noise and offset input voltage noise charactrizes as shown in the below table.

The dynamic range of the detector setup is $=20 \log$ $(2.5 \mathrm{~V} / 20.42 \mathrm{uV})=101.9 \mathrm{~dB}$. fig. 6 shows the printed circuit board of preamplifier system which composed of transimpedance amplifier(the MAX4206 chip) ,AVR ATmega microcontroller. The function of microcontroller is to control the grating movement as well as analog to digital converter data acquistion circuit.

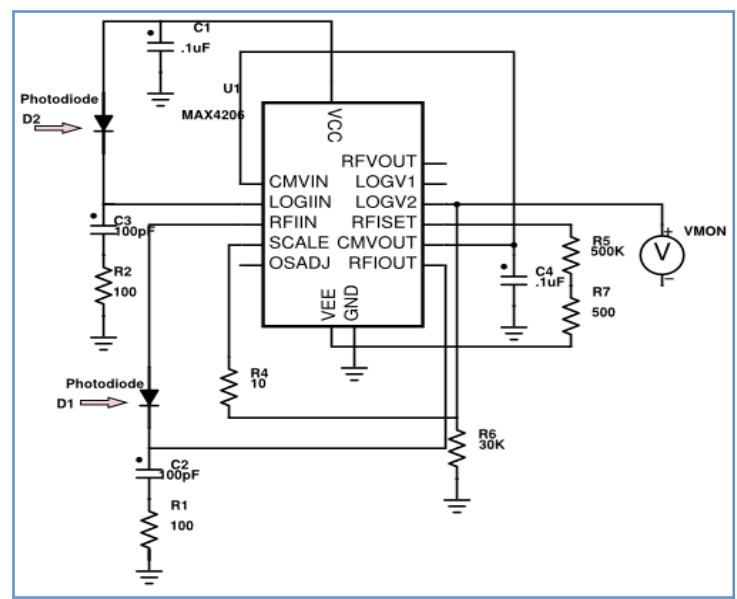

Figure 5. Photodiode amplifier circuit based on transimpedance amplifier.

TABLE I.

SHOWS THE DIFFERENT VALUES OF NOISES ON THE SETUP

\begin{tabular}{|l|c|}
\hline \multicolumn{1}{|c|}{ Type of noise } & Values $(\boldsymbol{\mu V \boldsymbol { r m s } )}$ \\
\hline Johnson & 6.40 \\
\hline Input voltage & 19.39 \\
\hline Output total & 20.426 \\
\hline
\end{tabular}

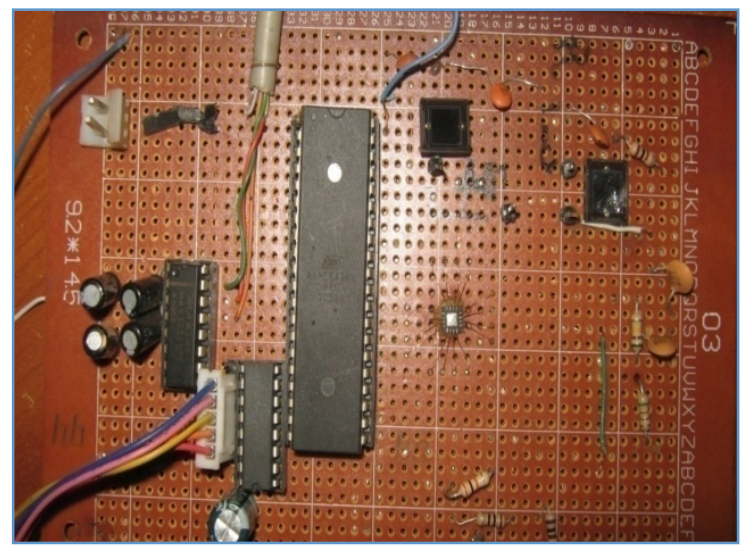

Figure 6. Printed circuit board of the transimpedance amplifier

\section{B. Sallen key low pass filter}

Filter design based on Butterworth characteristic it is output flat, but the phase response not linear. The design based on the sallen key low pass filter second order, the circuit is shown in fig.7. The selection of op amp for a filter based on two main factors: Gain bandwidth (GBW) and Slew rate, theses specifications are available in the op amp CA313, the selection of the other components based on the following values: $\mathrm{fc}=3.12 \mathrm{kHz} \mathrm{R} 1 / \mathrm{R} 2=0$. 586 $\mathrm{RA}=\mathrm{RB}, \mathrm{CA}=\mathrm{CB}$ and damping factor $=1$.

\section{RESUlts AND Discussions}

In this section, key parameters for validate the detector circuits is to verify the linearity of the sensor (photodiodes) output current and transimpedance amplifier output voltage and frequency response (transfer function) are tested. Furthermore, the developed system is used to run a typical experiment to detect the RNA in a sample.

\section{A. Photodiode and amplifier circuit}

The linearity of photodiodes sensors and transimpedance amplifier output voltage is tested by increasing the intensity of light gradually and measuring the corresponding output voltage. Fig. 8 shows the relation between the photodiode output current and the output voltage from the amplifier. Clearly the fig.8 evident that, the increasing of the light intensity incident on the photodiode lead to generate of more current according to photoelectric effect phenomena and the output voltage increased linearly.

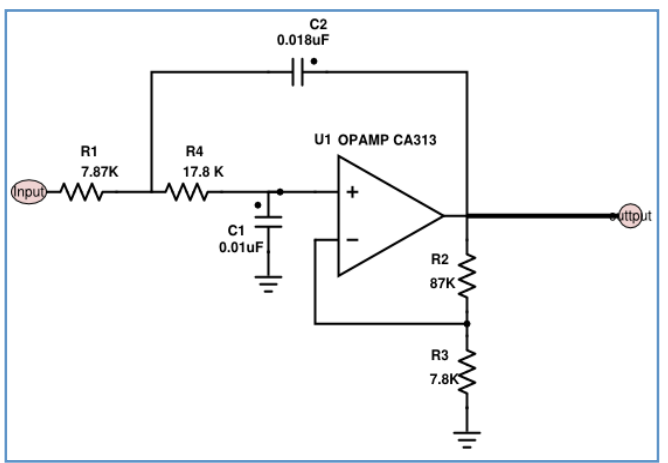

Figure 7. low pass sallen key filter

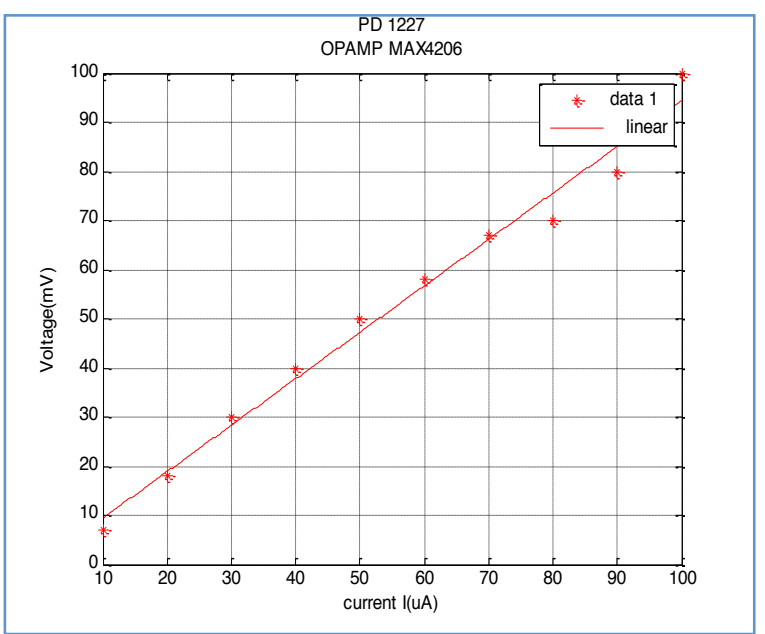

Figure 8. The relation between output current of the photodiode and the output voltage for the transimpedance amplifier circuit. 


\section{B. Frequency response analysis using transfer functions}

To characterize the frequency response, to see the advantage and the benefits of the feedback control system for the transimpedance amplifier circuit, Bode plot of magnitude and phase of the frequency reponse of the transimpedance amplifier circuit is shown in fig 9. The constant values used to plot the system response are given in table 2

TABLE II.

THE COMPONENTS OF THE TRANSIMPEDANCE AMPLIFIER FREQUENCY RESPONSE

\begin{tabular}{|c|c|c|}
\hline Amplifier & Source & Feedlback \\
\hline $\begin{array}{c}A=37500 \\
f=5 \mathrm{MHz}\end{array}$ & $C_{I N}=160 \mathrm{pF}$ & $R_{f}=30 \mathrm{k} \Omega$ \\
\hline
\end{tabular}

From the table2, $A$ represent the gain of the amplifier, $\mathrm{C}_{\mathrm{IN}}$ internal capacitance of the photodiode and $\mathrm{R}_{\mathrm{f}}$ is the feedback resistor.

$$
\operatorname{Tn} r(s)=R s h /(R s h+R s) \times R_{f} / s C j R s h+1
$$

Where $R s h / / R s$ and $C j$ is the parallel equivalent resistance and capacitance of the silicon photodiode[6].

Equation 3 described by Hernadez in [6] is used to obtain the output transfer functions $T n r$ (equation 4)and $T r$ (equation 5) respectively.

$$
\operatorname{Tn} r=\frac{30000}{1.6 e-06 s+1}
$$

The $\operatorname{Tr}$ of the transimpedance and the photodiode is given below

$$
\operatorname{Tr}=\frac{-9.333 e-05 s-25}{3.669 e-11 s^{2}+2.453 e-05 s+1}
$$

Fig. 9 shows the Bode diagram of the transfer functions of the transimpedance amplifier circuit detector obtained by plotting equation 4 and 5 . The result depicts that the frequency response of the detector circuit depends on the feedback resistor and the feedback capacitor, this affecting bandwidth.

\section{Application of the designed setup for the RNA analysis}

The important step in preparation of the nucleic acid, it is concentration and purity, nucleic acid absorbs light at $260 \mathrm{~nm}$ and $280 \mathrm{~nm}$ due to aromatic bases within their structure [15]. The measurement of RNA made using our double spectrophotometer as shows in the graph in Fig.10. Sample volume is $100 \mu \mathrm{L}$, sample blanked on water using a quartz cell $1 \mathrm{~cm}$, the blank water has been read, and then the RNA, the reading of the blank subtracted from the RNA sample to obtain absorbance of RNA.

From the fig. 11,the white vertical line bars indicate the presence of RNA in the sample and the upper vertical line bar indicates the presence of DNA in a sample.

\section{CONCLUSION}

In this work simplified double beam spectrophotometer is developed and successfully validated. Results on the test sample showed that the transimpedance amplifier and photodiodes can be used to build a simplified detector for the double beam spectrophotometer with excellent results.

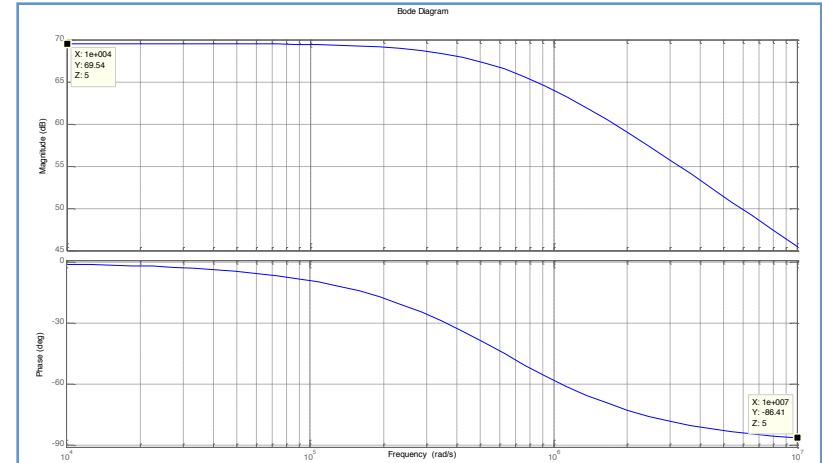

Figure 9. bode diagram of the transfer function of detector circuit based on the transimpedance amplifier circuit.

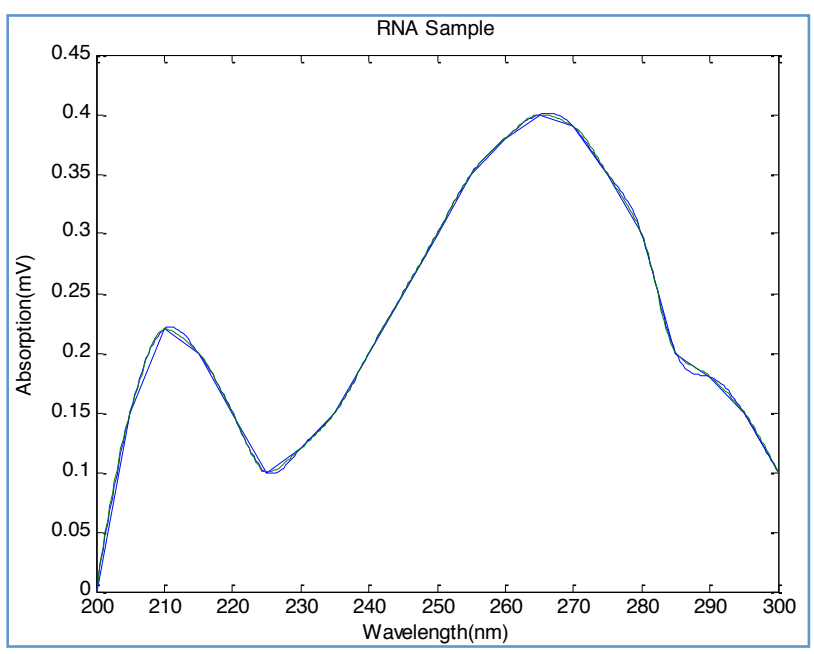

Figure 10. The absorbance of RNA sample

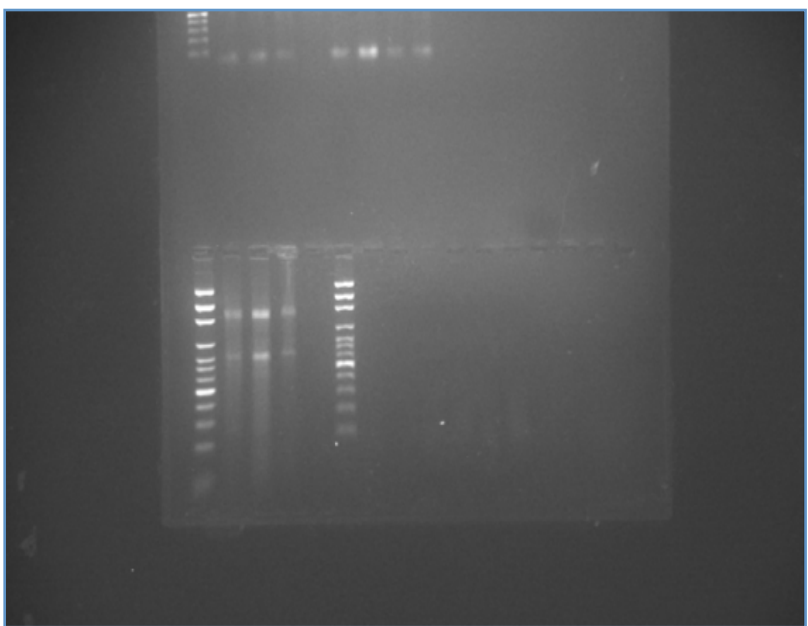

Figure 11. Picture of RNA sample using a garose gel electrophorsis and gel documentation system

As shown in fig. 10 , the peak present in $270 \mathrm{~nm}$ clearly indicates the presence of RNA in the sample.The purity assessment is determined using A260/A280 ratio for RNA and DNA are 2 and 1.8 respectively, in fig 10 the graph the ratio is 1.9 which indicate the present of RNA in the sample and reflect that the sample is contaminated. To ensure that our system has correctly detected the presence of RNA in the sample, the same sample is run on agrose a gel electrophosis and visualized it by using a gel documentation system as seen the fig. 11 . 
PAPER

Design of A Simple UV Double Beam SPectrophotometer Detector Based on A High Gain...

\section{REFERENCES}

[1] Kit, S., "Nucleotides and nucleic acids. Metabolic pathways", 4: p. 69-275, 1970.

[2] Lodish, H., A. Berk, and S. Zipursky, "Molecular cell biology" 4th edition, 2000.

[3] Cella, L.N., et al.,'Detection of RNA viruses: current technologies and future perspectives", Critical Reviews ${ }^{\mathrm{TM}}$ in Eukaryotic Gene Expression,2013.

[4] Datasheet, "Precision transimpedance logarithamatic amplifier with 5 decades of dynamic range,"Maxim,2003.

[5] Graeme, J," photodiode amplifiers: op amp solutions", McGrawHill,inc 1995.

[6] Hernandez,W, "Input-output transfer function analysis of a photometer circuit based on an operational amplifier.", Sensors 8(1): 35-50,2008. http://dx.doi.org/10.3390/s8010035

[7] Hernandez,W,"Photometer circuit based on positive and negative feedback compensations" ,Sensor Letters 5(3), 2007. http://dx.doi.org/10.1166/s1.2007.232

[8] Khandpur," Handbook of analytical instruments", Tata McGrawHill Education,2007.

[9] Moghimi, R, "Seven Steps To Successful Ultralow Light Signal Conversion".

[10] Orozco, L,"Programmable-Gain Transimpedance Amplifiers Maximize Dynamic Range in Spectroscopy Systems.", A Tribute to Jerry Fishman: 11,2013.

[11] Sobon,G.,P.R.Kaczmarek,et al, "High power fiber-based Femtosecond CPA System at 1560 NM.", Selected Topics in Electronics, IEEE Journal of 20(5): 492-496,2014. http://dx.doi.org/10.1109/JSTQE.2014.2301021

[12] Jones, C., B. Matthews, et al."Fabrication and assessment of optically immersed CdHgTe detector arrays.", Semiconductor Sci$\begin{array}{llll}\text { ence and Technology 6(12C): C110,1991. } & \text {. }\end{array}$ http://dx.doi.org/10.1088/0268-1242/6/12C/022
[13] Kester, W., S.Wurcer,et al" Section 5 High Impedance Sensors", Analog Devices, Inc.

[14] Alakhib,Abdelrahim,"Design of a low cost single beam spectrophotometer for DNA measurement."IJIRSET,Vol 5, issue 3,March 2016.

[15] O.Warburg and W.Christian,"Isolierung und Kristallisation des Gärungsferments Zymohexase", Naturwissenschaften, vol. 30, pp.731-731.

[16] A.Beer, "Bestimmung der Absorption des rothen Lichts in farbigen Flüssigkeiten", Annalen der Physik, vol. 162. ,pp. 78-88, 1852. http://dx.doi.org/10.1002/andp.18521620505

[17] Maxim,"integrated DC logarithamatic amplifier", AN-3611.

[18] Levine, P, Gong, P, Levicky, R, Shepard, "K. Active CMOS sensor array for the electrochemical Biomolecular detection", IEEE J.Solid-State Circuits, 43, 1859-1871,2008. http://dx.doi.org/10.1109/JSSC.2008.925407

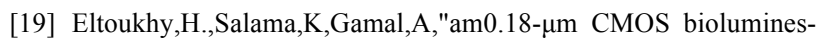
cence detection lab-on-chip", IEEE J. Solid-state Circuits, 41, 651-662,2006. http://dx.doi.org/10.1109/JSSC.2006.869785

[20] 1.Choi,J,Beaucage,G,Nevin,J,Lee,J,Puntambekar,A,Lee,J,"Dispos able smart lab on a chip for point-of-care clinical diagnostic", Proc. IEEE, 92.

\section{AUTHORS}

Alakhib Ibrahim Abdelbary is with Central Laboratory, Khartoum, Sudan.

Abdelrahim Elobied Ahmed is with Sudan International University, Khartoum, Sudan.

Submitted 02 May 2016. Published as resubmitted by the authors 05 June 2016. 